\title{
Missing children appeal designs: Is recall accuracy influenced by the design of the appeal?
}

\author{
Daniel Hunt ${ }^{1}$ (D) $\cdot$ Maria loannou $^{1} \cdot$ John Synnott $^{1}$ \\ Published online: 13 August 2019 \\ (C) The Author(s) 2019
}

\begin{abstract}
When a child goes missing it is common practice to release an appeal of the child in the hope that a member of the public could help to identify and locate them. Despite being an everyday occurrence, there remains a significant gap in our understanding of how effective these appeals are. The present study sought to explore the effectiveness of missing children appeals through the recall accuracy of the general public immediately after observing the appeals and again after a three-day delay. One hundred and eighty-two participants observed either a mock Child Rescue Alert or a mock Twitter appeal. The results found no significant difference in recall accuracy between the design of the appeals although there was a significant difference in recall error. Confidence in own recall accuracy and the length of time spent observing the appeals were also found to be significantly associated with recall accuracy. Initial recall accuracy scores were significantly higher than recall accuracy scores following a three-day break. This exploratory study demonstrates the requirement to improve missing children appeals and lays the foundation for future studies to build on these findings further.
\end{abstract}

Keywords Child rescue alert $\cdot$ Missing children $\cdot$ Missing persons $\cdot$ Recall accuracy $\cdot$ Twitter appeal

\section{Introduction}

There are an estimated 340,000 missing person incidents received annually in the United Kingdom by the police, the equivalent to one report every 90 seconds (Missing People 2018; National Crime Agency [NCA] 2017). Of these annual reports, approximately $60 \%$ relates to missing children making the United Kingdom generate one of the highest international rates for reports of missing children (European Commission 2013; NCA 2017). When a child goes missing it is commonplace for charities, law enforcement, family and friends of the child, to release photographs and details of the child through the media in the hope that members of the public can help to locate or identify the child (Lampinen et al. 2012a; Sweeney and Lampinen 2012). Releasing images and

Electronic supplementary material The online version of this article (https://doi.org/10.1007/s12144-019-00403-w) contains supplementary material, which is available to authorized users.

Daniel Hunt

D.Hunt2@hud.ac.uk

1 Department of Psychology, University of Huddersfield, Queensgate, Huddersfield HD1 3DH, UK descriptions of the missing child through the media allows the information to be seen by numerous individuals within a short period of time which wouldn't be possible using offline approaches (Fyfe et al. 2014; Taylor et al. 2013). Despite their importance and frequent use, however, there is a limited understanding on how effective this approach is (Drivsholm et al. 2017; Lampinen and Moore 2016; Lampinen et al. 2012b; Sweeney and Lampinen 2012). Therefore, the current study will build on this gap in the literature by exploring the effectiveness of missing children appeal designs on the recall accuracy and recall error of the general public.

The use of the media to release images and descriptions of missing children is not a novel approach. Media appeals for information have been disseminated by a variety of offline and online approaches such as milk cartons, websites, newspaper appeals, television broadcasts, posters, and text messages to name a few (Drivsholm et al. 2017). One of the most widely used and recognised approaches for distributing missing children appeals are through social media sites with an estimated $71 \%$ of appeals being distributed this way (Missing Children Europe n.d.). Social media sites allow appeals to be seen by an unlimited number of people within a short period of time due to the ability to 're-share' appeals. An example of this effect derives from the National Centre for Missing and Exploited Children ([NCMEC] 2015) whose appeal for information on 
an unknown and abandoned missing baby was viewed over 50 million times within a short period of time. Despite the clear advantages for using social media sites to disseminate appeals, there are some concerns relating to who is likely to be given media attention which may subsequently influence the likelihood of that child being located by the public quickly and prior to the child experiencing harm (Min and Feaster 2010).

The second most widely used approach for disseminating missing children appeals are through rescue alerts such as the Child Rescue Alert in the UK (Lampinen and Moore 2016; NCA 2014) and the America Missing Broadcast Emergency Response ([AMBER]; Gier et al. 2012) alerts in America. Rescue alerts are designed to issue state-wide alerts for abducted and non-abducted missing children who may appear to be in imminent danger or risk of serious harm (NCA 2014; U.S. Department of Justice 2015). The use of rescue alerts has a great advantage over other online methods of dissemination as they can retract the alerts upon the location of the missing child which is not always the case with online approaches (Drivsholm et al. 2017). However, with an estimated 200 AMBER alerts released annually, some have criticised the use of rescue alerts asserting that they are more of a "crime control theatre" than an essential tool (Griffin and Miller 2008, p.160; Griffin et al. 2007).

\section{Use of Photographs and Facial Recognition}

One of the major factors associated with the effectiveness of missing children appeals relies partly on the individual's ability to be able to correctly identify the missing child from the photograph (Gier et al. 2017; Mian and Mondloch 2012). However, this ability has been shown to be relatively poor with individuals typically only able to recognise faces that they are familiar with and not with unfamiliar faces which are common within missing children appeals (Burton et al. 1999; Davis and Valentine 2009; Megreya and Burton 2006, 2008). For instance, Sweeney and Lampinen (2012) explored the effect of missing children appeals on the facial identification of 96 student participants who were randomly assigned to observe an appeal with one image of the child or with three different images of the same child. Participants were then presented with 50 photographs of children and told to place them into two equal 'teams' and to alert the authorities using their computer keyboard if they came across the missing child (Sweeney and Lampinen 2012). The results found that the participants who had observed three images of the missing child had significantly greater identification accuracy than the participants who had only observed one photograph (Sweeney and Lampinen 2012).

Despite the increased accuracy for presenting multiple images of the same child, this effect is only prevalent when the images are of the same child. Lampinen and Moore (2016) randomly assigned 465 participants to observe three different mock missing adult appeals across a three-day period or to observe one missing adult appeal on the final day of the three-day event. The participants were told that the adult within the appeal would be seen around the university campus during the week and was to report the individual to the researchers if they located him (Lampinen and Moore 2016). The results found that the participants who had observed the single appeal had significantly greater identification accuracy than the participant who had observed three different appeals (Lampinen and Moore 2016).

The poor ability to identify faces from photographs is also demonstrated by the work of White et al. (2014) who explored the ability of 30 passport officers to identify and recognise faces from passport photographs. Thirty-four individuals were given either a genuine passport or a fraudulent passport which bore the photograph of an individual with similar physical appearances to the holder (White et al. 2014). The results found that $6 \%$ of genuine passports were incorrectly rejected by the passport officers whilst $14 \%$ of fraudulent passports were wrongly accepted (White et al. 2014). Hence, the human ability to recognise faces appear to be relatively poor particularly when there is just one image of an individual which can be observed.

\section{Use of Descriptions}

A second major factor associated with the effectiveness of missing children appeals derives from the written descriptions of the missing child which relates to their appearance and details of why the child may have gone missing. The influence of the written descriptions of missing children have not yet been explored and thus presents a significant gap in our understanding. Cary and Reder's (2003) work present an insight into this effect through their analysis of word recognition. Thirty student participants were presented with a list of single noun words that ranged from 16 words to 64 words on the list and were required to memorise as many of these words as possible. The participants were then presented with a recognition task and required to identify the words that were present on the list they had observed at the start of the experiment from a variety of single words (Cary and Reder 2003). The results found that the participants who had observed the shorter 16-word lists had significantly greater recognition accuracy than the participants who had observed the longer 64word lists (Cary and Reder 2003).

\section{Time Duration and Recall Ability}

The length of time that is spent observing missing children appeals may influence the effectiveness of that appeal for improving recall accuracy of the details enclosed. In principle, an increase in the amount of time available for the observation of an item allows the ability to encode this information to also 
increase which would maximise the potential for accurate retrieval (Kapardis 2014). This effect is demonstrated by the work of Yarmey et al. (2002) whereby members of the public were approached by a confederate asking for directions for five seconds or for $30 \mathrm{~s}$. The individuals were then approached by the researchers and were asked to recall details of the confederate (Yarmey et al. 2002). The results demonstrated that the individuals who had engaged with the confederate for just five seconds had significantly less accuracy in recall than the individuals who had engaged with the confederate for $30 \mathrm{~s}$ (Yarmey et al. 2002).

Likewise, Horry et al. (2014) explored the effect of time duration on recall accuracy via their analysis of archival eyewitness statements that were cross-referenced with video footage available of the crimes. Horry et al. (2014) found that the eyewitnesses who had been exposed to the different crimes for a greater period of time had significantly greater recall accuracy than the eyewitnesses who had only been exposed to the crimes for a shorter period of time. In relation to missing children appeals, Lampinen et al. (2012b) argue that time plays a significant role in the ability to encode and subsequently retrieve information. If there is only a limited amount of time available to observe the information in or is willing to be given by the individual themselves, then an increase in the number of items required to be observed results in the reduction of time available per item to be encoded (Lampinen et al. $2012 \mathrm{a}, \mathrm{b})$. This effect would reduce the likelihood of accurately recalling the information enclosed within the appeal.

\section{The Present Study}

The exploration of the effectiveness of missing children appeals is limited with only a handful of researchers investigating this (Drivsholm et al. 2017; Lampinen and Moore 2016; Lampinen et al. 2012b; Lampinen and Sweeney 2014; Sweeney and Lampinen 2012). In addition, research consistently suggests that members of the public are generally quite poor at being able to identify faces that are unfamiliar to them (Davis and Valentine 2009; Megreya and Burton 2006, 2008). This effect increases when the individuals' attention is elsewhere (Lampinen et al. 2012b; Miles 2005; Nobel and Shiffrin 2001). Therefore, the present study sought to fill in these gaps by exploring the effectiveness of missing children publicity appeals on the recall ability of the information enclosed within the appeals. The originality of the current study derives from the novel insight into how effective missing children publicity appeals are in being able to generate a high level of recall accuracy from members of the public. Similarly, the study also explores the effect of the design of missing children appeals on recall ability of members of the general public following a short three-day interval delay.

The study objectives are fivefold. First, to determine whether the length of time that is spent by an individual observing an appeal influences their subsequent recall accuracy and recall error scores. Second, to establish whether there are any significant differences in recall accuracy of the information enclosed between the type of design of the appeals and between the photograph-based and the text-based sections of the appeal. Third, to ascertain whether the level of confidence in the participants' own recall ability is associated with the overall recall accuracy and recall error scores. Forth, to determine if there are significant differences between the type of design of the appeal and the overall recall accuracy and recall error scores following a short three-day interval delay. Finally, the study further aims to explore whether there are significant differences between the initial recall accuracy and recall error scores with the delayed recall accuracy and recall error scores.

\section{Method}

\section{Design}

The current study adopted a mixed within-between subjects experimental design. The independent variables comprised of the type of appeal design with two levels: Child Rescue Alert design or Twitter Appeal design; and the level of confidence in own recall accuracy ranging from $0 \%$ confident to $100 \%$ confident. The dependent variables within the study comprised of the recall accuracy score recorded between $0 \%$ accurate and $100 \%$ accurate, the recall error score recorded between $0 \%$ error and $100 \%$ error, and the time duration spent observing the appeal calculated in seconds.

\section{Sample}

A total of 243 participants had completed the study using a mixed convenience and snowball sampling approach, although 61 participants were removed from the final data due to failing to complete the full study or misreading the task instructions. Therefore, the final sample comprises of 182 participants (151 females; $M_{\text {age }}=25.72$ years, $S D=11.61$ ) who were included in the analysis for the initial study results. Of the final 182 participants, $90(49.50 \%)$ had been randomly assigned to the Child Rescue Alert design condition and 92 $(50.50 \%)$ had been randomly assigned to the Twitter appeal design condition.

Following the initial experimental study, a secondary follow-up delayed experimental recall study was performed three days later. Of the original 182 participants who completed the initial experimental task, $71(39.01 \%)$ participants had dropped out and did not complete the follow-up recall task. Therefore, the final follow-up experimental study sample comprised of 111 participants who were included within the follow-up study results. The participant conditions for the delayed experimental study comprised of $56(50.50 \%)$ 
participants within the Child Rescue Alert design condition and $55(49.50 \%)$ participants within the Twitter appeal design condition.

All participants were recruited voluntarily via social media sites or the university's online recruitment system. Participants who took part in the study through social media did not receive any incentives for taking part although participants who took part in the study through the university's recruitment system received 0.5 credits. As the study was accessible online by the Qualtrics software, participants were free to take part in the study in any location and at any time of the day that they felt most comfortable with by accessing the study on their home computer or mobile device. The study was granted full ethical approval by the School Research Ethics Panel at the university. Table 1 demonstrates the demographic background statistics for the sample.

\section{Materials}

The participants completed one initial experimental study and one follow-up delayed recall task three days later. The present study comprised of the following.

\section{Fictional Missing Children Appeals}

Participants were randomly allocated to one of the two fictional missing children appeal designs: Child Rescue Alert design or Twitter appeal design. These designs were developed by the primary researcher to mimic real examples of missing children appeals. The Child Rescue Alert design enclosed an image of a fictional missing female child displayed on the left-hand side of the appeal. The right-hand side of the appeal enclosed textual information of the child that includes the missing child's name, the reason for going missing, physical appearance, height, and clothing last known to be wearing. The Twitter appeal design enclosed the same image of the fictional missing female child as well as the name and last known location of the child. The Twitter appeal design further included a tweet from Greater Manchester Police which includes identical information to the Child Rescue Alert design and stated: "can you help us find Abigail Walters from \#Salford? Went missing due to argument and last seen getting in the car of unknown male". There were no time limits for the two experimental conditions to be observed by the participants. The appeals were timed in the background by the Qualtrics software to distinguish the length of time each participant had spent observing the appeal which was not visible to the participants. The two appeal designs are presented in Fig. 1.

\section{Memory Distraction Task}

During the initial experimental study, participants were required to complete a short memory task to prevent rehearsal
Table 1 Demographic factors of ethnicity, education, employment, and marital status for the final sample

\begin{tabular}{|c|c|c|}
\hline Demographic Variable & $n$ & $\%$ \\
\hline \multicolumn{3}{|l|}{ Ethnicity } \\
\hline White British & 111 & 61.00 \\
\hline White Other & 15 & 8.20 \\
\hline Black & 8 & 4.40 \\
\hline Asian & 34 & 18.70 \\
\hline Chinese, Japanese, or Southern Asian & 1 & 0.50 \\
\hline Middle Eastern & 8 & 4.40 \\
\hline Other & 8 & 4.40 \\
\hline \multicolumn{3}{|l|}{ Highest Level of Education Completed } \\
\hline Primary & 1 & 0.50 \\
\hline Secondary & 8 & 4.40 \\
\hline College/Sixth Form & 117 & 64.30 \\
\hline Trade, Technical or Vocational & 6 & 3.30 \\
\hline Associate Degree & 6 & 3.30 \\
\hline Bachelors Degree & 24 & 13.20 \\
\hline Masters Degree & 14 & 7.70 \\
\hline Doctorate Degree & 3 & 1.60 \\
\hline Other & 3 & 1.60 \\
\hline \multicolumn{3}{|l|}{ Current Employment Status } \\
\hline Full Time & 24 & 13.20 \\
\hline Part Time & 58 & 31.90 \\
\hline Unemployed & 4 & 2.20 \\
\hline Retired & 1 & 0.50 \\
\hline Student & 124 & 68.10 \\
\hline Unable & 3 & 1.60 \\
\hline Other & 4 & 2.20 \\
\hline \multicolumn{3}{|l|}{ Marital Status } \\
\hline Single & 154 & 84.60 \\
\hline Married & 18 & 9.90 \\
\hline Widowed & 1 & 0.50 \\
\hline Divorced & 1 & 0.50 \\
\hline Separated & 1 & 0.50 \\
\hline Other & 7 & 3.80 \\
\hline
\end{tabular}

The cumulative percentages may be above $100 \%$ due to participants having the option to select more than one response

of the information enclosed within the appeals. The participants were unable to move forward to the next task or go back to the previous task for the duration of the memory distraction task. The memory distraction task presented the participants with five individual tables containing two columns and five rows. Each individual table enclosed ten single and randomised nouns which had been generated randomly by an online random word generator (www.randomlists.com/ nouns). Each individual table was displayed to the participants for $20 \mathrm{~s}$ and was presented in sequential order. Therefore, the distraction task totalled 50 nouns being presented for $100 \mathrm{~s}$. The participants were asked to "try and 
Any information? Please contact

Local Neighbourhood Policing Team

\section{ABIGAIL WALTERS}

Missing From: Bolton

Date: Tuesday $13^{\text {th }}$ July 2017 at 17:00

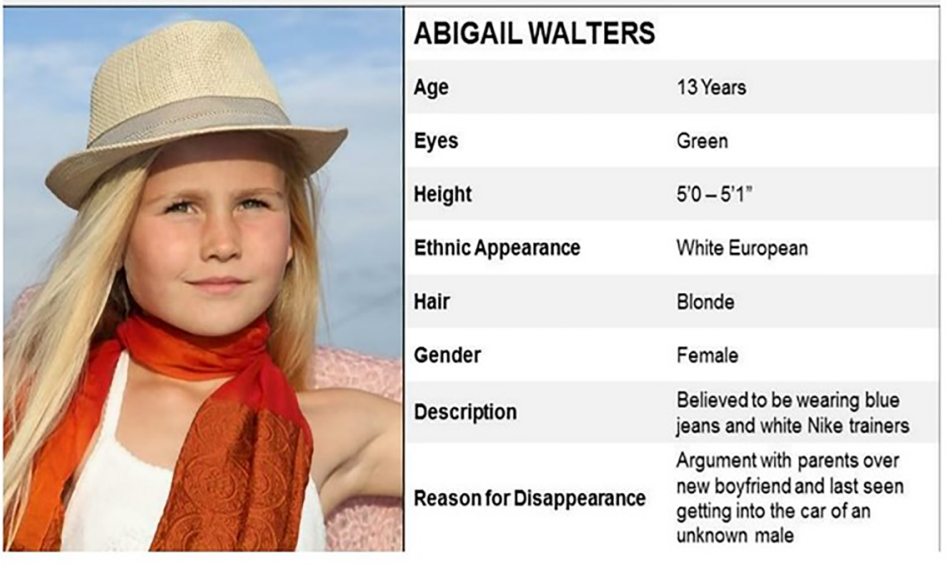

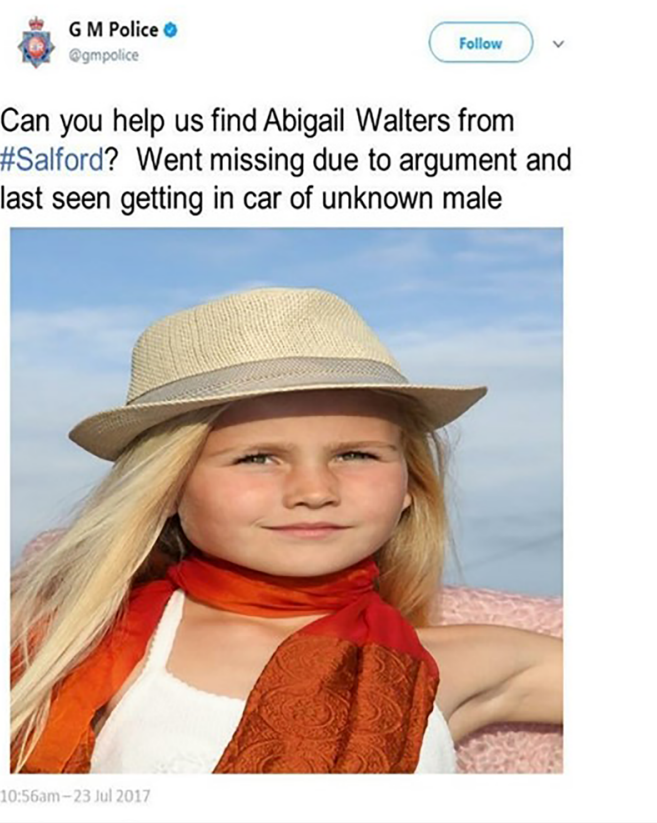

Fig. 1 Missing child appeal designs

remember as many of the words that will be displayed as possible as this may be tested later in the study". None of the participants were informed of this short distraction task prior to commencing the task.

\section{Free Recall Task}

The free recall task comprised of a single question and an open-field text box which requested participants to "try to write down as many of the details that you can remember from the missing child appeal you had observed at the very start of the study". The participants were also requested to indicate their level of confidence in their own recall accuracy on a slidable scale from $0 \%$ confident to $100 \%$ confident.

\section{Demographic Survey}

The final segment of the initial experimental study presented the participants with a short demographic survey. Participants were requested to include their current age, gender, ethnic background, the highest level of education completed, current employment status, and current marital status. Each of the demographic questions allowed the participants to select their responses from a list of pre-determined answers excluding age which was open-ended.

\section{Delayed Recall Task}

The delayed free recall task was identical to the recall task presented during the initial experimental study. Participants were asked to "try to write down as many of the details that you can remember from the missing child appeal you had observed three days ago at the very start of the previous study". Participants were once again requested to indicate their level of confidence in their recall accuracy following the three-day delay on a slidable scale that ranged from $0 \%$ confident to $100 \%$ confident.

\section{Procedure}

The experimental study was created using the Qualtrics Inc. survey software (www.Qualtrics.com). Participants who had observed the study details through social media or within the university's online recruitment system were requested to click the web link if they wished to take part. Participants were directed to an information sheet which detailed the experimental study's purpose, data collection and storage procedures, ethical considerations, and contact details of the researcher. Once the participants had read and understood the information enclosed, they were presented with a consent form. The Qualtrics software had then randomly assigned the participants into one of the two available experimental conditions where they were able to observe the Child Rescue Alert design or the Twitter appeal design.

Once the participants had finished observing the missing child appeal, they were presented with a short memory distraction task to minimise the likelihood of rehearsing the information enclosed within the appeals. Participants were requested to try and memorise as many nouns presented as possible. After $100 \mathrm{~s}$ when the distraction task ended, participants were redirected to the free recall task and were asked to try and write down as much of the details that they can remember 
from the information enclosed within the missing child appeal they had observed at the start of the study. The study was then concluded with the demographic survey, details of the delayed recall task, a request for an email address to participate in the delayed recall task, and a full debrief on the initial study.

Following a three-day break, the participants who had provided an email address were emailed to thank them for their participation in the first study and were presented with details of the follow-up study. Participants were presented with an individualised web link by Qualtrics which allowed their data submissions on the delayed recall task to be able to be matched up to their data submissions following the initial recall task. When the participants clicked on the web link they were directed to a brief information page which detailed the delayed recall task and a consent form. Once consent was provided, participants were presented with a free recall task that was identical to the one presented during the initial study. Participants were requested to try and write down as many details as possible that they could remember from the information enclosed within the missing child appeal they had observed three days previously at the start of the initial study. The participants were also requested to indicate their level of confidence in their own recall accuracy from a slidable scale which ranged from $0 \%$ confident to $100 \%$ confident. The delayed recall study ended with a full debrief of the full study and to thank them for their participation in the studies.

\section{Analysis}

Data were input manually by the primary researcher into SPSS version 24 for analysis. Recall accuracy scores were calculated by summing the number of correct items recalled by the participants and dividing this figure by the maximum number of potential items that were able to be recalled. This figure was then multiplied by 100 to derive a recall accuracy percentage score. Likewise, recall error scores were calculated by summing the number of incorrect items recalled by the participants and dividing this number by the total number of potential items able to be recalled. This number was multiplied by 100 to derive a recall error percentage score.

It is important to note that a recalled item was deemed to be correct if it was identical to the information within the appeal whilst the absence of a target item was not included in the calculation of the recall error score. For instance, if a participant indicated that the missing child had blonde hair, this would be classified as a correct recalled item and would contribute to the participant's recall accuracy score. In contrast, if the participant had stated the missing child had brunette hair, this would be classified as an incorrect recalled item and would contribute to the participant's recall error score. If the participant did not include any details for the colour of the missing child's hair, this did not contribute to either the recall accuracy score or the recall error score.

The study also sought to explore factors associated with the details of the missing child enclosed within the photograph and within the text in the appeals during the participant recall tasks. Participant recall responses relating to details included in the photograph such as the missing child wearing a red scarf, a hat, and a white top were included within the analysis for photograph-based details. Participant recall responses relating to details within the text such as the height of the missing child, location missing from and reason for going missing were included within the analyses for textual-based details. The full items available to be recalled can be seen in the appendix. All remaining variables were dichotomous in nature and were coded as $0=$ not present or $1=$ present except age which was open-ended.

\section{Results}

\section{Descriptive Statistics}

Descriptive statistics for the mean length of time spent in seconds by the participants observing the missing children appeals, mean confidence percentage scores in own recall accuracy, and mean overall recall accuracy and recall error percentage scores during the initial experimental task is displayed in Table 2. Descriptive statistics for the number of correct and incorrect items recalled by participants observing the Child Rescue Alert design and the Twitter appeal design for the initial and follow-up studies can be viewed in the appendix.

Table 2 Descriptive statistics of the mean time in seconds spent by participants observing the appeals, mean confidence percentage, mean recall accuracy scores, and mean recall error scores of the initial experiment

\begin{tabular}{|c|c|c|c|c|c|c|c|c|c|c|c|c|c|c|c|c|}
\hline \multirow[t]{2}{*}{ Experimental Condition } & \multicolumn{4}{|c|}{ Time (s) } & \multicolumn{4}{|c|}{ Confidence $(\%)$} & \multicolumn{4}{|c|}{ Recall Accuracy (\%) } & \multicolumn{4}{|c|}{ Recall Error (\%) } \\
\hline & $m$ & $S D$ & Min & Max & $m$ & $S D$ & Min & Max & $m$ & $S D$ & Min & $\operatorname{Max}$ & $m$ & $S D$ & Min & Max \\
\hline Child Rescue Alert Design & 25.32 & 19.08 & 2.48 & 137.85 & 63.69 & 25.05 & 9 & 100 & 35.77 & 17.68 & 4.76 & 80.95 & 2.06 & 2.68 & .00 & 9.52 \\
\hline Twitter Appeal Design & 15.29 & 14.74 & 1.05 & 105.83 & 57.71 & 26.81 & 5 & 100 & 34.53 & 18.69 & .00 & 76.92 & 1.59 & 3.52 & .00 & 15.38 \\
\hline
\end{tabular}




\section{Initial Experiment}

\section{Time Duration}

The first objective sought to explore if the length of time that was spent by the participants when observing the missing children appeals influenced the recall accuracy and recall error percentage scores. A Spearman's rho correlation found a large and positive monotropic relationship between the length of time spent by the participants and their overall recall accuracy score when observing the Child Rescue Alert design $\left(r_{s}=.68, n=90, p=<0.001\right)$. A secondary Spearman's rho correlation found a moderate and positive monotropic association between the length of time spent by the participants and their overall recall accuracy score when observing the Twitter appeal design $\left(r_{s}=.45, n=92, p=\right.$ $<0.001)$. There were no significant associations between the length of time spent by the participants and their overall recall error scores for those observing the Child Rescue Alert design $\left(r_{s}=0.52, n=90, p=0.63\right)$ or the Twitter appeal design $\left(r_{s}=-0.03, n=92, p=0.76\right)$.

\section{Format Design}

The second objective sought to determine whether there were significant differences in recall accuracy scores and recall error scores between the type of design observed by participants. A Mann Whitney U test found no significant difference between the overall recall accuracy scores and the type of design observed $(U=3865.00, z=-0.78, p=0.44, r=$ -0.06). Participants who had observed the Child Rescue Alert design were found to have marginally higher recall accuracy scores $(M d n=33.33$, Mean Rank $=94.56)$ than participants who had observed the Twitter appeal design $(M d n=$ 69.50, Mean Rank $=88.50$ ). In contrast, there was a significant difference found between overall recall error scores and the type of design observed $(U=3510.00, z=-2.22, p=0.03$, $r=-0.16)$ with participants who observed the Child Rescue Alert design having significantly greater recall error scores $(M d n=0.00$, Mean Rank $=98.50)$ than participants observing the Twitter appeal design $(M d n=0.00$, Mean Rank $=84.65)$.

The research further sought to explore if the difference between the type of appeal design observed influenced the overall recall accuracy and recall error scores based upon the type of information enclosed within the appeal. The first Mann Whitney U test sought to explore whether there were significant differences between the type of design observed and the overall recall accuracy and recall error scores of the photograph information enclosed. The results found a significant difference $(U=3380.00, z=-2.20, p=0.03, r=-0.16)$ with participants observing the Child Rescue Alert design having significantly greater recall accuracy on information from the photograph $(M d n=33.33$, Mean Rank $=99.94)$ compared to participants observing the Twitter appeal ( $M d n$ $=16.67$, Mean Rank $=83.24$ ). There was no significant difference between the type of design observed and the overall recall error scores for photograph information enclosed $(U=$ $3755.00, z=-1.68, p=0.09, r=-0.12$ ).

A second Mann Whitney $U$ test sought to explore whether there were significant differences between the two appeal designs and the overall recall accuracy scores for information enclosed within the text only. The results found a nonsignificant difference $(U=4439.00, z=0.84, p=0.40, r=$ $0.06)$ with participants observing the Twitter appeal design having marginally higher recall accuracy scores $(M d n=$ 42.86, Mean Rank $=94.75$ ) than participants observing the Child Rescue Alert design $(M d n=38.89$, Mean Rank $=$ 88.18). In contrast, the results found a significant difference between the two appeal designs and overall recall error scores for information enclosed within the text $(U=3072.00, z=$ $-4.09, p=<0.001, r=-0.30$ ). Participants who observed the Child Rescue Alert design had significantly higher recall error scores $(M d n=0.00$, Mean Rank $=103.37)$ than participants who observed the Twitter appeal design $(M d n=0.00$, Mean Rank $=79.89)($ Fig 2).

\section{Confidence}

The fourth objective of the study sought to explore whether confidence in recall accuracy was associated with the overall recall accuracy scores derived from the free-recall task. A Spearman's rho correlation was performed finding a significant and positive association $(r=.64, n=90, p=$ $<0.001)$ between the level of confidence in own recall accuracy and the overall recall accuracy score for participants who observed the Child Rescue Alert design. Higher levels of confidence $(M=63.69, S D=25.05)$ were associated with higher recall accuracy scores $(M=35.77, S D=17.68)$. A secondary Spearman's rho correlation was performed to explore whether the level of confidence in own recall accuracy was associated with overall recall accuracy scores for participants observing the Twitter appeal, finding a significant relationship ( $r=.61, n=92, p=<0.001)$. Participants who indicated higher confidence in their own recall accuracy $(M=57.71, S D=26.81)$ were associated with having higher recall accuracy scores $(M=34.53, S D=18.69)$.

\section{Delayed Experiment}

\section{Time}

In addition to the exploration above, the study further sought to explore whether the time spent by participants observing the initial appeal three days earlier influences the overall delayed recall accuracy scores. The Mann Whitney U test found that there was not a significant difference between time spent 
Fig. 2 Photograph-based and Text-based mean recall accuracy and recall error scores for child rescue alert design and twitter appeal design

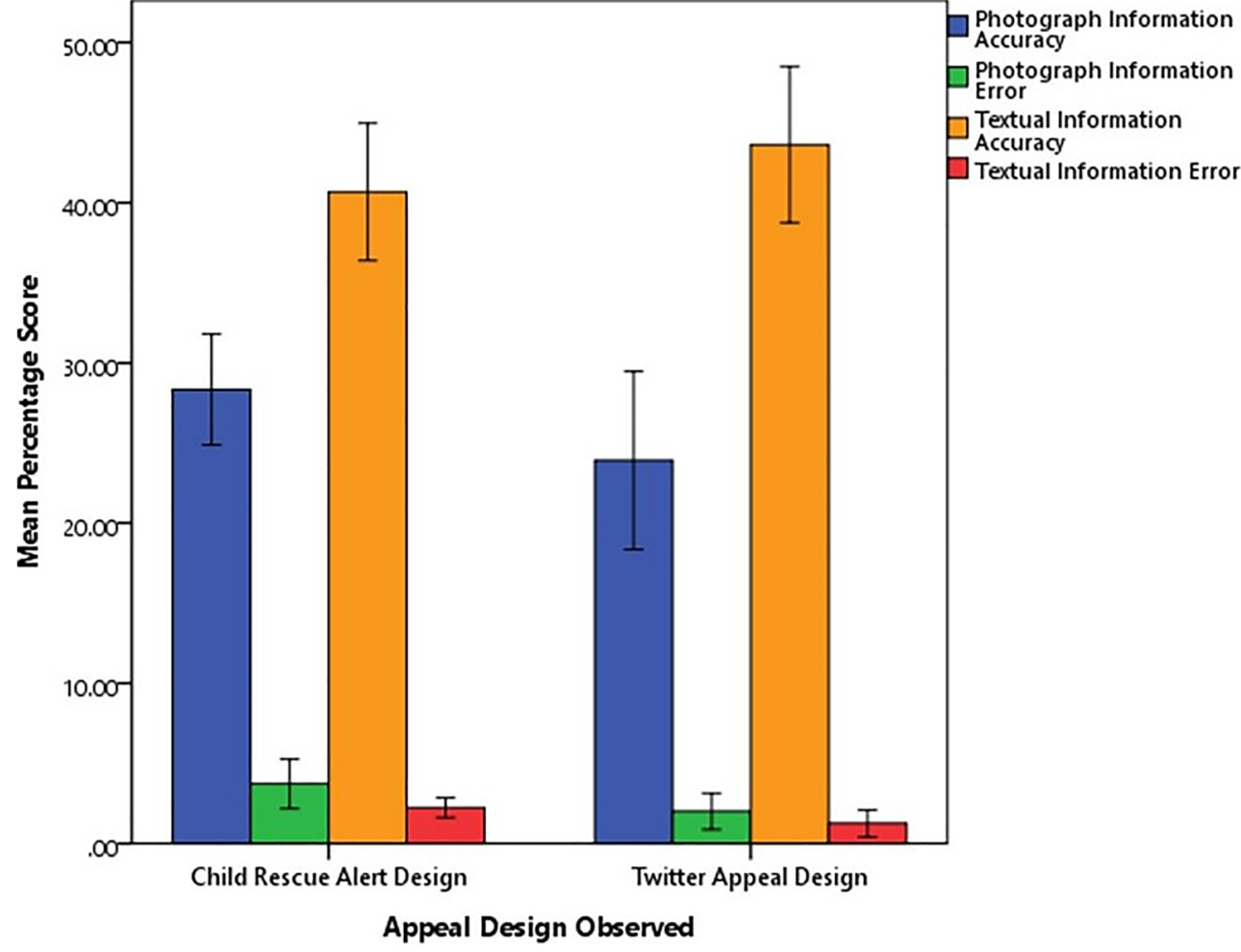

and recall accuracy $(U=1541.00, z=0.01, p=0.99, r=0.09)$ with participants scoring a near identical mean recall accuracy score between the observation of the Child Rescue Alert design $(M d n=26.19$, Mean Rank = 55.98) and the Twitter appeal design $(M d n=23.08$, Mean Rank $=56.02)$.

\section{Format Design}

To determine if the type of appeal design influences the overall recall accuracy following a three-day delay, a Mann Whitney $U$ test was performed. The results found a nonsignificant difference $(U=1541.00, z=0.01, p=0.99, r=$ $0.09)$ with participants observing the Child Rescue Alert design having similar recall accuracy scores $(M d n=26.19$, Mean Rank $=55.98)$ to participants observing the Twitter appeal design $(M d n=23.08$, Mean Rank = 56.02). In contrast, a secondary Mann Whitney U test found a significant difference between appeal design and overall recall error scores following a three-day delay $(U=1136.00, z=-2.88, p=0.004, r=$ -0.27). Participants who had observed the Child Rescue Alert design had greater recall error scores $(M d n=0.00$, Mean Rank $=63.21)$ than participants who observed the Twitter appeal design $(M d n=0.00$, Mean Rank $=48.65)$.

The research further sought to explore the effects of the type of information enclosed within the appeal on the overall recall accuracy and recall error scores following a three-day delay. For information recalled from the photograph enclosed, the results found a non-significant difference $(U=1385, z=$ $-0.95, p=0.34, r=-0.09)$ with participants observing the
Child Rescue Alert design having similar recall accuracy scores $(M d n=16.67$, Mean Rank $=58.77)$ to participants observing the Twitter appeal design $(M d n=16.67$, Mean Rank = 53.18). The results also found a non-significant difference between the types of appeal designs for recall error scores following a three-day delay $(U=1499, z=-0.38, p=$ $0.71, r=-0.04$ ).

In relation to the recalled information from participants following a three-day delay on the textual details enclosed within the appeal designs, the results found a significant difference $(U=867.50, z=-3.99, p=<0.001, r=-0.38)$. Participants observing the Child Rescue Alert design had significantly higher recall accuracy scores following a three-day delay $(M d n=27.78$, Mean Rank $=68.01)$ than participants who had observed the Twitter appeal design $(M d n=16.67$, Mean Rank =43.77). Additional analyses found that there was also a significant difference in recall error scores between the two appeal designs following a three-day delay $(U=$ 836.00, $z=-5.22, p=<0.001, r=-0.50)$. Participants who had observed the Child Rescue Alert had higher recall error scores $(M d n=0.00$, Mean Rank $=66.57)$ than participants who had observed the Twitter appeal design $(M d n=0.00$, Mean Rank = 42.77).

\section{Confidence}

To explore whether there are associations between the level of confidence in the participants' own recall and the overall recall accuracy score following a three-day delay, a series of 
Spearman rho correlations were performed. The results found a significant and positive association between the level of the participants' confidence and their overall recall accuracy scores for participants observing the Child Rescue Alert appeal design $\left(r_{s}=.55, n=56, p=<0.001\right)$ and for participants observing the Twitter appeal design $\left(r_{s}=.59, n=55, p=\right.$ $<0.001$ ). Additional analyses found that there were no significant associations between the level of confidence in the participants' accuracy and the overall recall error scores for both the Child Rescue Alert Design $\left(r_{s}=0.16, n=56, p=0.23\right)$ and the Twitter appeal design $\left(r_{s}=-0.06, n=55, p=0.66\right)$.

\section{Initial and Delayed Recall Association}

The final objective of the study sought to explore the differences between initial recall accuracy and recall error scores with the delayed recall accuracy and recall error scores. The repeated measures ANOVA for recall accuracy scores found a significant main effect $\left(F(1,109)=58.35, p=<0.001, n_{p}^{2}=\right.$ $0.349)$. There were significantly higher overall recall accuracy scores during the initial recall task $(M=36.83, S D=17.40)$ compared to the overall recall accuracy scores following a three-day delay $(M=27.01, S D=15.38)$. The results also found that there was not a significant main effect between the initial recall accuracy scores and the delayed recall accuracy scores between the two appeal designs $(F(1,109)=0.06$, $\left.p=0.813, n_{p}^{2}=0.001\right)$. A second repeated measures ANOVA was performed to explore the differences between the initial recall error scores and the delayed recall error scores following a three-day delay. The results found a non-significant main $\operatorname{effect}\left(F(1,109)=1.07, p=0.304, n_{p}^{2}=0.010\right)$ (Fig. 3).

\section{Discussion}

The aim of the study was to explore the effectiveness of missing children appeals on recall accuracy. The study sought to determine whether the type of design for the missing child appeal influenced the overall recall accuracy and recall error for the information enclosed. The results indicate that the type of design does have some influence on the overall recall accuracy and recall error of the information enclosed although some of the associated factors were not significant.

\section{Effect of Time}

The study findings suggest that the time duration spent observing a missing child appeal did have a significant association with recall accuracy irrespective of the type of the design. Participants who had observed the appeal for a greater duration of time were significantly more likely to have higher recall accuracy than participants who had spent less time observing the appeals. This result could be explained via the ability to fully maximise the encoding processes of the observed information from the short-term memory to the longterm memory which subsequently improves the ability to
Fig. 3 Overall mean recall accuracy and recall error scores between the Child Rescue Alert design and the Twitter appeal design for initial and follow-up recall tasks

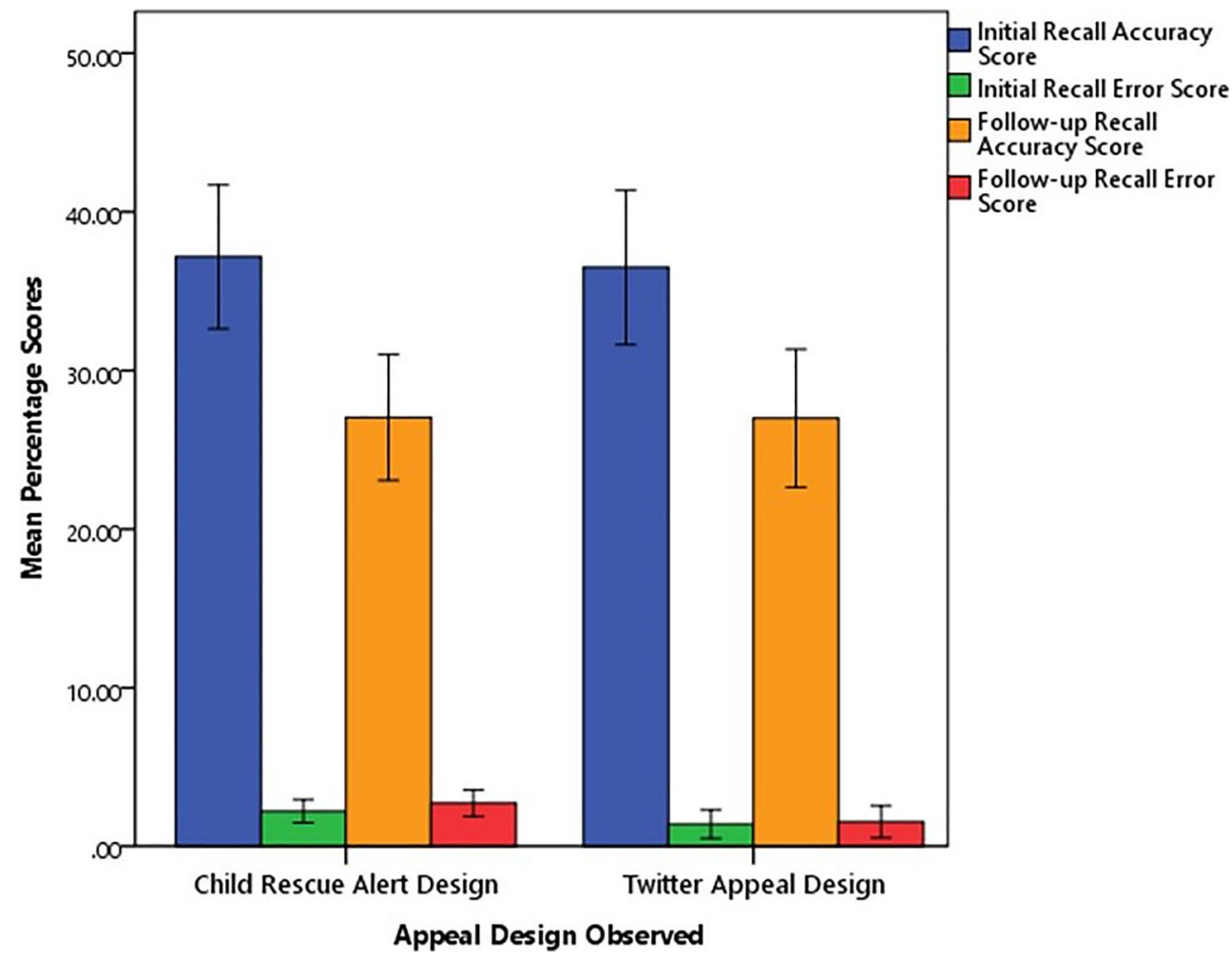


retrieve more information in a more accurate manner (Kapardis 2014). Moreover, the results support the previous findings by Horry et al. (2014) who analysed archival eyewitness statements of crimes and found that the eyewitnesses who had been exposed to the offender for a greater period of time had significantly higher identification accuracy than the eyewitnesses who had only been exposed to the offenders for a shorter period of time.

The findings are also supported by the work of Yarmey et al. (2002) who sought to explore the effect of time on the recall ability of a stranger. Members of the public had been exposed to a confederate asking for directions for five seconds or for $30 \mathrm{~s}$ and were then approached to recall the confederate's physical characteristics Yarmey et al. (2002). Their findings found that the individuals who had spent $30 \mathrm{~s}$ with the confederate had significantly higher recall accuracy than the individuals who had only spent five seconds with the confederate (Yarmey et al. 2002). Therefore, in relation to missing children appeals, the longer the duration of time that an individual consumes or is willing to consume to the appeal, the higher their recall accuracy would be (Lampinen et al. 2012a, b).

\section{Effect of Design}

The study further sought to explore the effect of the type of design of the missing children appeals on the overall recall accuracy but did not find any significant differences. There was, however, a significant difference between the Child Rescue Alert design and the Twitter appeal design on the recall accuracy of information recalled from the enclosed photograph. Participants who had observed the Child Rescue Alert design were found to have significantly greater recall accuracy on details enclosed within the photograph compared to participants who had observed the Twitter appeal design.

Additional analyses also found significant differences in the overall recall error with individuals observing the Child Rescue Alert design having higher recall error scores than individuals observing the Twitter appeal design. Recall error scores for details enclosed within the text-based information within the appeals were also found be significantly different. Individuals observing the Child Rescue Alert design had significantly higher recall error scores than individuals observing the Twitter appeal design. This finding could be explained due to the additional text enclosed within the Child Rescue Alert appeal which required additional encoding compared to the Twitter appeal that enclosed a smaller number of textual information.

These findings thus fall in line with Cary and Reder (2003) who aimed to explore the effect of the length of words on recall ability. Participants were assigned to different conditions whereby they were presented with a range of single word nouns ranging in length from 16 words to 64 words (Cary and
Reder 2003). Once observation of the lists of words had ended, participants were presented with a recognition test and asked to identify the words that were present on their own list (Cary and Reder 2003). The results found that individuals who had observed just 16 words had significantly greater recall accuracy than individuals who had observed the longer list of 64 words (Cary and Reder 2003). Therefore, increasing the number of words required to be encoded and recalled reduces the recall accuracy and increases the recall error as supported by the current findings.

The current findings had also found a significant difference between the type of design of the missing child appeal and the photograph that was enclosed with individuals observing the Child Rescue Alert appeal having significantly higher recall accuracy than individuals observing the Twitter appeal. This finding presents an additional novel insight as the photograph was identical across both format designs but was more prominent in the Twitter appeal taking up most of the available space compared to the Child Rescue Alert which had the image presented for only half of the appeal. Consequently, the size of the photograph may influence the likelihood of greater recognition and encoding which future research could explore further. Nevertheless, the current study demonstrates that higher recall accuracy of a missing child's photograph is significantly more likely to occur via the observation of a Child Rescue Alert design than a Twitter alert design.

\section{Effect of Confidence}

The third research objective sought to explore whether an individual's level of confidence in their own recall ability influences the overall recall accuracy. The results suggest that there is a significant and positive association with participants who indicated higher levels of confidence in their own recall also acquiring higher recall accuracy percentage scores. In contrast, confidence was not significantly associated with recall error. This finding supports the work of Memon et al. (2003) who examined the identification accuracy of 164 participants and found those with a higher level of confidence in their own identification accuracy had significantly greater overall identification accuracy than participants who indicated a lower level of confidence. Likewise, Brewer and Wells (2006) found that from a sample of 1200 individuals, those who had indicated higher levels of confidence in accuracy had significantly greater accuracy than those who indicated a low level of confidence. However, as the current literature exploring the confidence-accuracy association is mixed (Goodsell et al. 2009; Kassin et al. 2001; Wright and Stroud 2002), the current results must be read with caution. 


\section{Effect of Delay}

The final objective of the study was to explore the effect of a short three-day interval between observation of an appeal and recalling the information enclosed. The results suggest that participants who had higher recall accuracy scores initially also had significantly higher recall accuracy scores during the delayed recall task. Participants with higher initial recall error scores were also found to have higher delayed recall error scores following the short three-day interval. In addition, the results also indicate that the overall delayed recall accuracy scores were significantly lower compared to the initial overall recall accuracy scores regardless of the type of design of the missing children appeals.

Cary and Reder's (2003) work therefore partially supports this finding as their work exploring recall accuracy of varying lists of single words found those who had higher initial recall accuracy scores were also found to have higher recall accuracy scores following a mid-break distraction task. Although not exploring the recall accuracy following a long break, the study supports the current study's findings that initial higher recall accuracy is associated with higher recall accuracy following a delay in recall. In relation to missing children, it would be particularly uncommon for an individual to observe a missing child appeal and then immediately locate and identify the child. The location and identification of the child could take a matter of hours to a matter of days and thus, additional research would be highly advantageous to explore these effects further.

\section{Limitations and Future Directions}

Building on the limited understanding of the effectiveness of missing children publicity appeals, the current study presents a novel insight into some of the factors associated with the design of the appeal on recall accuracy immediately after observation and again following a short three-day delay. However, there are several limitations that must be addressed. First, the distraction task utilised within the initial study was included to prevent participants from rehearsing the information they have just observed by focusing their attention elsewhere via a memory task. As this task was a simple memory task and was only presented for two minutes, participants may not have been fully prevented from rehearsing the information which would have influenced the recall scores. Nonetheless, the three-day interval allowed participants to continue with their daily activities which would naturally prevent them from rehearsing the information within the appeals as participants were unaware that they would have to recall the information again in the follow-up task. Future research could, therefore, improve the initial study by utilising longer and more cognitively demanding distraction tasks.
Second, the study aimed to acquire a representative sample of the UK population although the sample consisted primarily of participants with a white background ethnicity, was female and were students. Therefore, future research could address this issue by repeating the study with a more representable sample to further explore the key factors associated with recall accuracy and gender, ethnicity, geographical location, employment status and level of education. Nevertheless, the study sample did comprise of participants who were within different geographical locations across the country and thus minimises the potential for biases due to the geographic location of individuals.

Finally, all the participants were free to participate in the study at any time of the day and in any location that they had wished due to the online design. This may prove consequential as participants were unable to be prevented from taking notes if they had wished during the task which would have significantly influenced the recall accuracy and error scores. However, the study aimed to replicable realistic events in daily activities and research has demonstrated how members of the public are unlikely to give $100 \%$ of their attention and concentration to every appeal presented (Miller et al., 2009). Similarly, these individuals may be distracted by completing alternative tasks at the same time as the appeal such as eating a family meal which would not have been possible to achieve in a laboratory-based environment with these factors strictly controlled.

\section{Conclusion}

The present study findings suggest that the design of the missing children appeals does have some effect on subsequent recall accuracy. Child Rescue Alert appeals were found to produce significantly higher recall errors than the Twitter alert appeal. There are no significant differences between the two designs for recall accuracy, although there are marginal differences. The results also found that the level of confidence in own recall accuracy is significantly associated with the overall recall accuracy as higher confidence levels were associated with higher recall accuracy scores regardless of the design of the appeal observed. Initial recall accuracy is also significantly associated with the subsequent recall accuracy following a short three-day interval.

These findings present significant implications for the current practice of disseminating missing children appeals as the design of the appeal has been found to influence the recall accuracy of the details enclosed. In addition, the length of time spent observing the appeals, the level of confidence in own recall ability, and the type of content enclosed within the appeals such as photograph or textual information are also found to significantly influence the rate of recall accuracy. The current findings are not to dispute the use of missing children appeals but to improve their effectiveness in helping to 
relocate missing children by improving the design of these appeals, potentially a standardised design of missing persons appeals that maximises the potential for greater recall accuracy and minimises the possibility of recall errors. However, as this study is exploratory, additional research is required to identify and improve the factors that are associated with greater recall accuracy that would subsequently improve the effectiveness of missing children publicity appeals.

\section{Compliance with Ethical Standards}

Conflict of Interest On behalf of all authors, the corresponding author states that there is no conflict of interest.

Ethical Approval All procedures performed within the study that involved human participants was in accordance with the ethical standards of the University of Huddersfield (SREP/2017/095) and with the ethical standards of the British Psychological Society Code of Ethics and Conduct (2018).

Informed Consent Informed consent was obtained from all individual participants included in the study

Open Access This article is distributed under the terms of the Creative Commons Attribution 4.0 International License (http:// creativecommons.org/licenses/by/4.0/), which permits unrestricted use, distribution, and reproduction in any medium, provided you give appropriate credit to the original author(s) and the source, provide a link to the Creative Commons license, and indicate if changes were made.

\section{References}

Brewer, N., \& Wells, G. L. (2006). The confidence-accuracy relationship in eyewitness identification: Effects of lineup instructions, foil similarity, and target-absent base rates. Journal of Experimental Psychology, 12(1), 11-30. https://doi.org/10.1037/1076-898x.12.1. 11.

Burton, M., Wilson, S., Cowan, M., \& Bruce, V. (1999). Face recognition in poor-quality video: Evidence from security surveillance. Psychological Science, 10(3), 243-248. https://doi.org/10.1111/ 1467-9280.00144.

Cary, M., \& Reder, L. M. (2003). A dual-process account of the listlength and strength-based mirror effects in recognition. Journal of Memory and Language, 49(2), 231-248. https://doi.org/10.1016/ s0749-596x(03)00061-5.

Davis, J. P., \& Valentine, T. (2009). CCTV on trial: Matching video images with the defendant in the dock. Applied Cognitive Psychology, 23(4), 482-505. https://doi.org/10.1002/acp.1490.

Drivsholm, M., Moralis, D., Shalev-Greene, K., \& Woolnough, P. (2017). Once missing never forgotten? Results of scoping research on the impact of publicity appeals in missing children cases. Retrieved from: https://researchportal.port.ac.uk/portal/files/7514950/Once Missing_Never_Forgotten_final.pdf. Accessed 22 June 2019.

European Commission. (2013). Missing children in the European Union: Mapping, data collection and statistics. Retrieved from: http://eu. europa.eu/justice/fundamental-rights/files/missing_children_study_ 2013_en.pdf. Accessed 20 May 2019.

Fyfe, N. R., Stevenson, O., \& Woolnough, P. (2014). Missing persons: The processes and challenges of police investigation. Policing and
Society: An International Journal of Research and Policy, 1-17. https://doi.org/10.1080/10439463.2014.881812.

Gier, V. S., Kreiner, D. S., \& Hudnell, W. J. (2012). AMBER alerts: Are school-type photographs the best choice for identifying missing children? Journal of Police and Criminal Psychology, 27(1), 9-23. https://doi.org/10.1007/s11896-011-9085-z.

Gier, V. S., Kreiner, D. S., \& Lampinen, J. M. (2017). Factors affecting recognition of senior citizens in a silver alert. Journal of Police and Criminal Psychology, 32(3), 185-196. https://doi.org/10.1007/ s11896-016-9210-0.

Goodsell, C. A., Neuschatz, J. S., \& Gronlund, S. D. (2009). Effects of mugshot commitment on lineup performance in young and older adults. Applied Cognitive Psychology, 23(6), 788-803. https://doi. org/10.1002/acp.1512.

Griffin, T., \& Miller, M. K. (2008). Child abduction, AMBER alert, and crime control theatre. Criminal Justice Review, 33(2), 159-176. https://doi.org/10.1177/0734016808316778.

Griffin, T., Miller, M. K., Hoppe, J., Rebideaux, A., \& Hammack, R. (2007). A preliminary examination of AMBER alert's effects. Criminal Justice Policy Review, 18(4), 378-394. https://doi.org/10. $1177 / 0887403407302332$.

Horry, R., Halford, P., Brewer, N., Milne, R., \& Bull, R. (2014). Archival analyses of eyewitness identification test outcomes: What can they tell us about eyewitness memory? Law and Human Behavior, 38(1), 94-108. https://doi.org/10.1037/lhb0000060.

Kapardis, A. (2014). Psychology and law: A critical introduction (4th ed.). New York: Cambridge University Press.

Kassin, S. M., Tubb, V. A., Hosch, H. M., \& Memon, A. (2001). On the "general acceptance" of eyewitness testimony research: A new survey of the experts. American Psychologist, 56(5), 405-416. https:// doi.org/10.1037/0003-066x.56.5.405.

Lampinen, J. M., \& Moore, K. N. (2016). Missing person alerts: Does repeated exposure decrease their effectiveness? Journal of Experimental Criminology, 12(4), 587-598. https://doi.org/10. 1007/s11292-016-9263-1.

Lampinen, J. M., \& Sweeney, L. N. (2014). Associated adults: prospective person memory for family abducted children. Journal of Police and Criminal Psychology, 29(1), 22-27. https://doi.org/10.1007/ s11896-013-9120-3.

Lampinen, J. M., Miller, J. T., \& Dehon, H. (2012a). Depicting the missing: Prospective and retrospective person memory for age progressed images. Applied Cognitive Psychology, 26(2), 167173. https://doi.org/10.1002/acp.1819.

Lampinen, J. M., Peters, C. S., \& Gier, V. S. (2012b). Power in numbers: The effect of target set size on prospective person memory in an analog missing child scenario. Applied Cognitive Psychology, 26(5), 702-708. https://doi.org/10.1002/acp.2848.

Megreya, A. M., \& Burton, A. M. (2006). Unfamiliar faces are not faces: Evidence from a matching task. Memory \& Cognition, 34(4), 865876. https://doi.org/10.3758/BF03193433.

Megreya, A. M., \& Burton, A. M. (2008). Matching faces to photographs: Poor performance in eyewitness memory (without the memory). Journal of Experimental Psychology: Applied, 14(4), 364-372. https://doi.org/10.1037/a0013464.

Memon, A., Hope, L., \& Bull, R. (2003). Exposure duration: Effects on eyewitness accuracy and confidence. British Journal of Psychology, 94(3), 339-354. https://doi.org/10.1348/000712603767876262.

Mian, J. F., \& Mondloch, C. J. (2012). Recognizing identity in the face of change: The development of an expression-independent representation of facial identity. Journal of Vision, 12(7), 1-11. https://doi.org/ 10.1167/12.7.17.

Miles, T. J. (2005). Estimating the effect of America's most wanted: A duration analysis of wanted fugitives. The Journal of Law \& Economics, 48(1), 281-306. https://doi.org/10.1086/428718. 
Miller, M. K., Griffin, T., Clinkinbeard, S. S., Thomas, R. M. (2009). The psychology of AMBER Alert: Unresolved issues and implications. The Social Science Journal, 46(1),111-123

Min, S., \& Feaster, J. C. (2010). Missing children in national news coverage: Racial and gender representations of missing children cases. Communication Research Reports, 27(3), 207-216. https://doi.org/ 10.1080/08824091003776289.

Missing Children Europe. (n.d.). Publicity Appeals. Retrieved from: http://missingchildreneurope.eu/publicityappeals. Accessed 22 June 2019.

Missing People. (2018). Key information. Retrieved from: https://www. missingpeople.org.uk/about-us/about-the-issue/research/76keyinformation2.html. Accessed 22 June 2019.

National Centre for Missing and Exploited Children. (2015). National centre for missing and exploited children annual report. Retrieved from: http://www.missingkids.com/content/dam/ncmec/en us/ publications/ncmec2015.pdf. Accessed 20 May 2019.

National Crime Agency [NCA] (2014). New alert system for missing children in danger. Retrieved from: http://www. nationalcrimeagency.gov.uk/news/368-new-alert-system-formissing-children-in-danger. Accessed 10 April 2019.

National Crime Agency [NCA]. (2017). Missing persons data report 2015/2016. Retrieved from: http://missingpersons.police.uk/en-gb/ resources/downloads/download/61. Accessed 22 June 2019.

Nobel, P. A., \& Shiffrin, R. M. (2001). Retrieval processes in recognition and cued recall. Journal of Experimental Psychology: Learning,
Memory, and Cognition, 27(2), 384-413. https://doi.org/10.1037// 0278-7393.27.2.384.

Sweeney, L. N., \& Lampinen, J. M. (2012). The effect of presenting multiple images on prospective and retrospective person memory for missing children. Journal of Applied Research in Memory and Cognition, 1(4), 235-241. https://doi.org/10.1016/j.jarmac.2012.08.001.

Taylor, J., Boisvert, D., Sims, B., \& Garver, C. (2013). An examination of gender and age in print media accounts of child abductions. Criminal Justice Studies, 26(2), 151-167. https://doi.org/10.1080/ 1478601x.2012.724683.

U.S. Department of Justice (2015). Guidelines for issuing AMBER alerts. Retrieved from: http://www.amberalert.gov/guidelines.htm. Accessed 22 June 2019.

White, D., Kemp, R. I., Jenkins, R., Matheson, M., \& Burton, A. M. (2014). Passport officers' errors in face matching. PLOSone, 9(8), 1-6. https://doi.org/10.1371/journal.pone.0103510.

Wright, D., \& Stroud, J. N. (2002). Age differences in lineup identification accuracy: People are better with their own age. Law and Human Behavior, 26(6), 641-654. https://doi.org/10.1023/A: 1020981501383.

Yarmey, A. D., Jacob, J., \& Porter, A. (2002). Person recall in field settings. Journal of Applied Social Psychology, 32(11), 23542367. https://doi.org/10.1111/j.1559-1816.2002.tb01866.x.

Publisher's note Springer Nature remains neutral with regard to jurisdictional claims in published maps and institutional affiliations. 